\title{
Adaptive Machine Learning Based Network Intrusion Detection
}

\author{
Hatitye Chindove \\ Dane Brown* \\ hatitye@gmail.com \\ d.brown@ru.ac.za \\ Rhodes University \\ South Africa
}

\begin{abstract}
Network intrusion detection system (NIDS) adoption is essential for mitigating computer network attacks in various scenarios. However, the increasing complexity of computer networks and attacks make it challenging to classify network traffic. Machine learning (ML) techniques in a NIDS can be affected by different scenarios, and thus the recency, size and applicability of datasets are vital factors to consider when selecting and tuning a machine learning classifier. The proposed approach evaluates relatively new datasets constructed such that they depict real-world scenarios. It includes analyses of dataset balancing and sampling, feature engineering and systematic ML-based NIDS model tuning focused on the adaptive improvement of intrusion detection. A comparison between machine learning classifiers forms part of the evaluation process. Results on the proposed approach model effectiveness for NIDS are discussed. Recurrent neural networks and random forests models consistently achieved high f1-score results with macro f1-scores of 0.73 and 0.87 for the CICIDS 2017 dataset; and 0.73 and 0.72 against the CICIDS 2018 dataset, respectively.
\end{abstract}

CCS Concepts: - Security and privacy $\rightarrow$ Intrusion detection systems; • Computing methodologies $\rightarrow$ Feature selection; Supervised learning by classification.

\section{ACM Reference Format:}

Hatitye Chindove and Dane Brown. 2021. Adaptive Machine Learning Based Network Intrusion Detection. In International Conference on Artificial Intelligence and its Applications (icARTi '21), December 9-10, 2021, Virtual Event, Mauritius. ACM, New York, NY, USA, 6 pages. https://doi.org/10.1145/3487923.3487938

${ }^{*}$ Both authors contributed equally to this research.

Permission to make digital or hard copies of all or part of this work for personal or classroom use is granted without fee provided that copies are not made or distributed for profit or commercial advantage and that copies bear this notice and the full citation on the first page. Copyrights for components of this work owned by others than ACM must be honored. Abstracting with credit is permitted. To copy otherwise, or republish, to post on servers or to redistribute to lists, requires prior specific permission and/or a fee. Request permissions from permissions@acm.org.

icARTi '21, December 9-10, 2021, Virtual Event, Mauritius

() 2021 Association for Computing Machinery.

ACM ISBN 978-1-4503-8575-6/21/12 ..\$15.00

https://doi.org/10.1145/3487923.3487938

\section{Introduction}

As the world has become more dependent on computer systems, the infrastructure has experienced an increase in the scale of security attacks [12]. A 2019 IBM report ${ }^{1}$ shows that successful attacks have an average detection time of 206 days, coupled with a 314-day life cycle to contain each security breach. As such, early detection can assist in improving response times for any system seeking to maintain a high-security posture [6]. It follows that intrusion detection is an essential part of monitoring computer events. A Network Intrusion Detection System (NIDS) is one such security tool that identifies an inside attack, outside attack and unauthorised access into a computer network.

NIDS generally use two methods for detection, namely, signature-based and anomaly-based detection. In signaturebased detection, pre-installed rules for the attacker patterns are made use of detect attacks. Anomaly-based detection uses traffic shape observations to measure the deviation from normal traffic [7]. Anomaly-based detection using machine learning (ML) has recently shown promising results [11].

A key characteristic of ML-based techniques is their ability to learn and improve their performance over time [14]. Common strategies for ML emphasise building a framework that enhances its execution based on previous results - changing execution strategy based on recently acquired data - to classify an intrusion. ML techniques have the advantage of adaptability and capturing of interdependencies when effectively implemented. However, an immediate challenge with ML is the general need to compromise between model complexity and training duration. Selecting appropriate algorithms for NIDS is vital in addressing this problem. Regardless of ML-based NIDS potential, there still are challenges and limitations associated with it that require comprehensive review in developing NIDS.

Additionally, in real-world systems, most network traffic is non-malicious; it follows that generally categorising malignant traffic may be challenging for NIDS. Hence, this paper aims to address the above challenges by developing ML-based multi-class NIDS effective on new/unseen data.

\footnotetext{
www.all-about-security.de/fileadmin/micropages

Fachartikel_28/2019_Cost_of_a_Data_Breach_Report_final.pdf
} 
Top-performing NIDS models are evaluated and contrasted against related systems found in the literature.

\section{Literature Review}

The following section describes supervised ML-based NIDS approaches - NIDS labelled by their ML algorithm - according to related work. Subsequently, datasets are reviewed to reflect the intended contributions.

\subsection{Related Work}

K-Nearest Neighbour (KNN) is an algorithm that assumes similarities exist at a close distance. The algorithm selects $k$ entries in a dataset that is closest to the new data point. A majority vote of the prevalent classes among those $k$ entries will be the class of the new data point. Atefi et al. [2] achieved a 0.964 accuracy with KNN on the CICIDS 2017 dataset.

Support Vector Machines (SVM) is an algorithm that first maps the input vector into a higher dimensional feature space and obtains an optimal hyperplane in the higher dimensional feature space [4]. The hyperplane aims to separate two classes of data points by finding the largest margin between two points. Aksu et al. [1] used SVM to determine the effect of each feature on classification. The findings show a selection of 30 features producing an f1-score of 0.656 .

Decision Trees (DT) is a technique based on a divide and conquer strategy, which utilises decision nodes and leaf nodes where a test over one of the attributes and a leaf node represents the class value [14]. Bisht and Ahmad [3] used DT and attained an f1-score of 0.831 .

Random Forests (RF) is an algorithm consisting of a large volume of individual DT's that operate as an ensemble [10]. Each tree in the RF outputs a class prediction. When combined, the class with the most votes becomes the model's prediction. Bisht and Ahmad [3] used RF for NIDS and achieved a $0.809 \mathrm{f} 1$-score, i.e lower than DT's accuracy.

Multi-layer Perceptrons (MLP) is a feed-forward Artificial Neural Network (ANN) that has a series of algorithms that recognise underlying relationships in a dataset [9]. Typically, back-propagation is used for training in a supervised manner. MLP is composed of activation functions that are chained together in a layer. The layers are fully connected as each unit in the layer is influenced by all previous units. Chuanlong et al. [5] utilised MLP in a multi-class classification NIDS and achieved an accuracy of 0.781 .

Recurrent Neural Networks (RNN) is an ANN technique that contains loops, allowing information storage within the network [9]. RNN also uses back-propagation learning through storing recurring time sequences. Chuan-long et al. [5] used an RNN model composed of 80 hidden nodes and a 0.1 learning rate. The model achieved an accuracy of 0.833 and 0.813 for binary and multi-class, respectively.

\subsection{Datasets}

This paper focused on evaluating realistic data from CICIDS $2017^{2}$ and CICIDS $2018^{3}$ datasets. These datasets reflect practical scenarios in composition and data distribution. The CICIDS 2017 dataset was gathered by Sharafaldin. et al. [13] using simulated attack and victim networks. The dataset was prelabeled by the authors of the dataset and adopted in this system. The output is statistical features calculated separately for a bidirectional network. The final dataset constitutes 83 statistical features and a classification label, each calculated for forward and reverse network traffic directions. The dataset is composed of 15 classes used in this paper.

The CICIDS 2018 dataset follows a similar data gathering methodology as the CICIDS 2017 but in a different setting. Context data of the attacks provided by the dataset authors for the defined scenarios and adopted in the best effort to label the CICIDS 2018 dataset using time windows. The dataset has some different attack scenarios from CICIDS 2017 and a distinct attacking infrastructure. The dataset is composed of 15 classes $^{4}$, but three of those classes are additional $D D o S$ variants. Many classes are similar to CICIDS 2017 but CICIDS 2018 lacks Heartbleed and PortScan.

\section{Proposed Methodology}

The proposed methodology ascertains the top-performing approaches for NIDS that are optimised for macro f1-score on class detection in the evaluated datasets. Each classifier undergoes much experimentation - based on the literature review - for this paper. Figure 1 shows the approach used for creating and evaluating ML-based NIDS in this paper.

The system goes through several stages in the pipeline, namely; 1) Data Acquisition, 2) Data Preparation, 3) Feature Engineering, and 4) Model Evaluation and Tuning. ML processing pipelines are adopted to prevent data leakage in the test harness by ensuring that data preparation is constrained to each fold of the cross-validation procedure.

\subsection{Data Acquisition}

During preliminary visualisation and testing for the CICIDS 2017 dataset, numeric features were loaded as floats and integers. Downcasting all data as integers except the 'Label' column, loading speeds significantly improved across the different datasets. This was necessary to address time constraints and memory limitations during experimentation without noticeable model effects.

The extracted CICIDS 2018 data was used as semantic information to determine different variants of classes - both known and unknown - for labelling, using rules from regular

\footnotetext{
${ }^{2}$ https://www.unb.ca/cic/datasets/ids-2017.html

${ }^{3}$ https://www.unb.ca/cic/datasets/ids-2018.html

${ }^{4}$ Benign, Bot, DDoS, DoS(GoldenEye, Hulk, Slowhttptest, slowloris), Patator(FTP and SSH), Heartbleed, Infiltration, PortScan, Web (Brute Force, Sql Injection and XSS)
} 


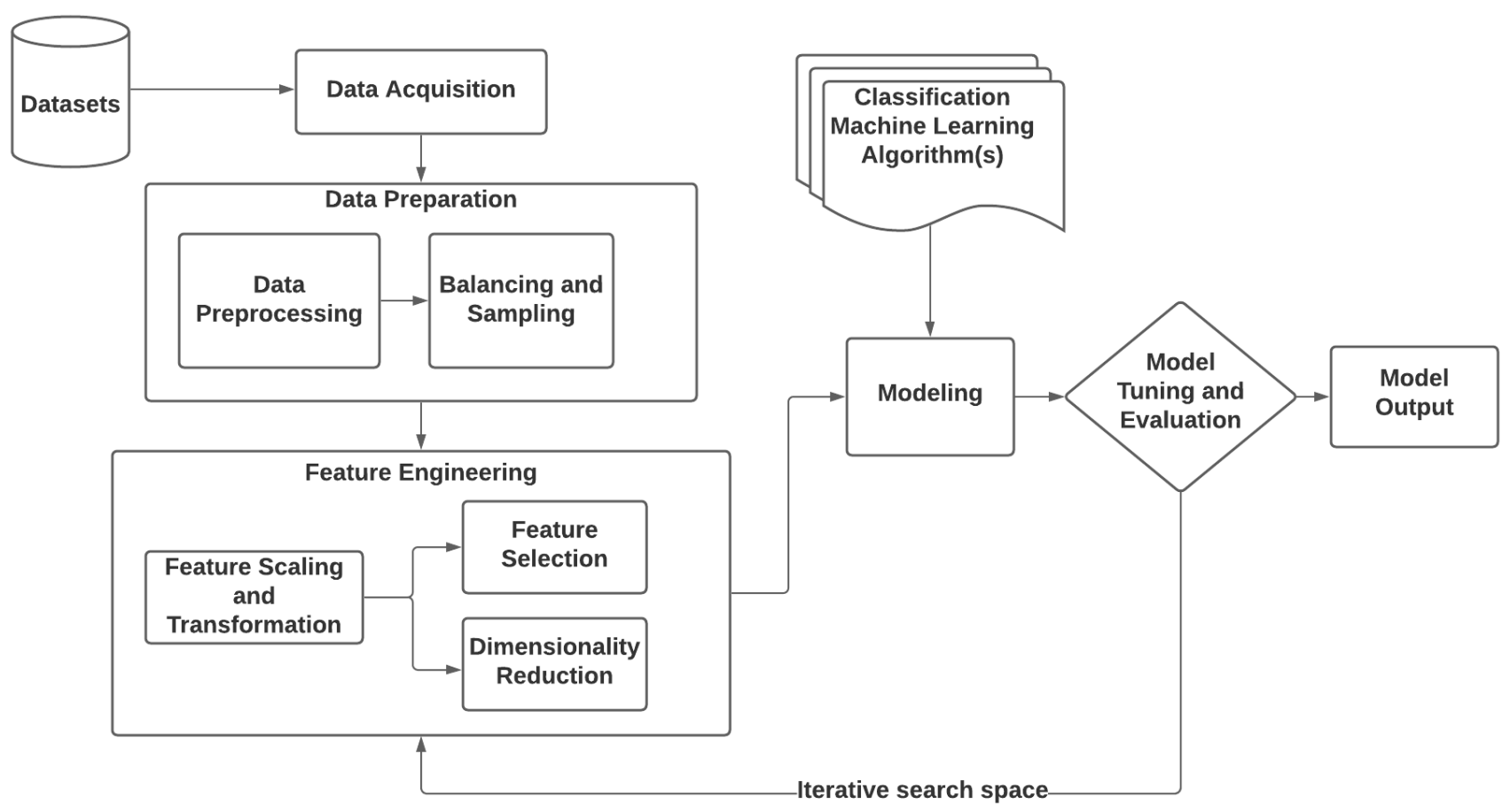

Figure 1. Experiment Design Overview.

expressions. The caveat of the approach is that sub-classes of attacks can be inaccurately labelled for unknown attacks in CICIDS 2018 - as was the case for the 3 DDoS variants. Furthermore, the CICIDS 2018 dataset includes some different attack scenarios from the CICIDS 2017 dataset.

\subsection{Data Preparation}

3.2.1 Data Preprocessing. Data was first merged into a single dataset by loading all the CSV files into data frames and consolidating them into a single data frame. Note that the first field is typically a header containing the feature names per column. After merging, it was discovered that there are duplicate header fields throughout the CICIDS 2017 CSVs. These were removed, resulting in a total of 2,660,377 samples.

Data samples were dropped that contained duplicate records or unrealistic features similar to that of Reis et al. [10] and Rosay et al. [11] - where it was argued that the data loss is necessary for accurate real-world application. This reduces the number of features to 69 usable features for the ML training process. Duplicates records were removed from the CICIDS 2017 validation set records. As part of the formatting, classes were encoded to have integer outputs rather than strings outputs.

3.2.2 Balancing and Sampling. Stratified sampling was applied to the dataset to separate training and test data. Poststrata, the sample is selected randomly in proportion with a seed of 42 for reproduction purposes. Training/test data splits of 10/90, 30/70 and 50/50 percentage were selected to observe the effect of the training data size on the models. $10 / 90$ was determined to be the minimum distributable train/test split ratio (limited data) with the assumption that if a model performs well with this limited data, it may perform well on unmodelled data. Furthermore, the use of this can reflect the effectiveness in more real-world scenarios.

There was a clear presence of a class imbalance in the CICIDS 2017 dataset. Minority classes were those with a representation of fewer than 5000 samples. Synthetic Minority Oversampling Technique (SMOTE) was validated by adding 100 to 500 samples to minority classes. Two hundred synthetic samples produced optimal results during preliminary tests but yielded insignificant changes to classification performance. Random Undersampling was subsequently applied to remove excess samples from majority classes if they contain more than 50000 samples. Of note, SMOTE was applied only to the training data for a fair comparison - avoiding data contamination during validation and the test set.

A preliminary test showed that contaminating the test data and adding synthetic instances to the original dataset made it possible to achieve a macro f1-score of 0.94 on a $10 / 90$ train/test split. However, this was excluded from the proposed implementation. Contaminating the validation set lead to perfect accuracy during parameter tuning but also severe overfitting. 


\subsection{Feature Engineering}

3.3.1 Feature Scaling and Transformation. Several feature scaling and transformation approaches are evaluated in the pipeline, where standardisation is applied to mitigate distance-based classifier problems. The Standard, Power, Quantile and Robust scaling techniques are evaluated in this step of the experiment. Note, non-distance-based classifiers - RF and DT - have cases where scaling/transformation is not applied due to their scale-invariant nature.

3.3.2 Feature Selection and Dimensionality Reduction. For feature selection, ranking techniques were used: Gini Importance (GI) and Permutation Importance (PI). These methods were evaluated across all scalers. Using the Python time module, calculating the PI function took $302.19 \mathrm{~s}$, whilst the GI function took 2.12 seconds. PI is adopted as a method that could be generally applied. PI ranks the best features in each model by permuting over 34 (features / 2) iterations and explicitly selecting features with the best f1-score for a particular base model.

Alternate to feature selection, dimensionality reduction using the Principal Component Analysis (PCA) method is adopted. Other subspace ${ }^{5}$ methods were tested on the validation set but not presented in the findings due to observed limitations, including the tendency to overfit, low classification performance, and high computational requirements. The first 26 principal components explain 0.99 of variance used to identify the number of required PCA components. Note, PCA transforms data into a vector (Eigen) space that can be scaled effectively for distance methods.

\subsection{Model Tuning and Evaluation}

The proposed method evaluates several supervised ML classifiers highlighted in Section 2. As a training strategy, One versus All (OvA) is used. 26PCA or 26PI - 26 components by PCA or 26 top-selected features by PI - represents the feature space reduction approach. Moreover, Q-26PCA-MLP represents a model application, where the first character represents the scaling method - Quantile as Q, Power as P followed by the feature space reduction method - 26PCA and finally the tuned classification algorithm - MLP.

While each ML classifier has diverse hyperparameter tuning parameters, Grid Search was used for an exhaustive search. A series of experiments were conducted for each tuned ML classifier against its best feature space reduction method. The findings are used for contrasting purposes of each output ML model against related systems.

The macro-average is the preferred metric because it gives all classes equal importance. It computes the f1 metric independently for each class and averages them, whereas a micro-average aggregates the contributions of all classes.

\footnotetext{
${ }^{5} \mathrm{LDA}$, LLE, t-SNE and UMAP
}

For unseen data, features extracted during data acquisition are used to evaluate CICIDS 2017 models' effectiveness. The CICIDS 2018 dataset is processed in the same way as the CICIDS 2017 dataset, but with the following difference to allow for automated reuse of a model on different data during classification: 1) Regular expression matching is used to select classes that are suspected to be similar to those already modelled on CICIDS 2017 in the previous experiments, leading to 13 classes. 2) Class labels are matched to the models' labels and included for subsequent classification of those specific labels.

The final experiment evaluates the classification performance of CICIDS 2018 by following the same hyperparameter tuning and training methodology as the first experiment. The classes that were grouped during the regular expression matching for unseen data are used for the remodelling.

\section{Results}

\subsection{Validation Set Model Results}

The model classifier parameter tuning process was initiated by finding the optimal features to adopt per base model through evaluating the CICIDS 2017 validation set. Following the feature selection step, classifier base models are tuned by ranking their respective PI according to the 69 feature set. PI and PCA are compared to determine which approach effectively reduces feature space and improves classification accuracy. In general, PI had varying effects per ML model. Results show that this step was vital for RF, DT, KNN and MLP classifiers. When performing additional dimensionality reduction, PCA produced better results compared to other explored extraction methods.

The hyperparameters are based on the bias/variance tradeoff by selecting the value where both training and crossvalidation scores peak. The best performing models using the limited data are analysed to prove efficacy against training data dependence. Multi-class classification models are trained for all the classes in the CICIDS 2017 dataset.

Table 1 shows the CICIDS 2017 best results for the NIDS models on limited data. 26PI-RF achieves the best macro f1-score of 0.87 with a precision as high as 0.90 .

Table 1. Best results for CICIDS 2017 limited data models.

\begin{tabular}{lcccc}
\hline Model & Accuracy & Precision & Recall & $\begin{array}{c}\text { f1-score } \\
\text { (macro) }\end{array}$ \\
\hline 26PI-RF & $\approx 1$ & 0.90 & 0.86 & 0.87 \\
Q-26PI-KNN & $\approx 1$ & 0.80 & 0.86 & 0.82 \\
Q-36PI-MLP & $\approx 1$ & 0.83 & 0.85 & 0.81 \\
Q-26PI-SVM & $\approx 1$ & 0.82 & 0.84 & 0.81 \\
16PI-DT & $\approx 1$ & 0.78 & 0.83 & 0.76 \\
Q-69PI-RNN & $\approx 1$ & 0.74 & 0.82 & 0.73 \\
\hline
\end{tabular}


26PI-RF yields the best results with limited data attaining a 0.87 macro f1-score plus a perfect (rounded) accuracy and micro f1-score, outperforming the 0.9648 accuracy achieved by Kurniabudi et al. [8], which was the best observed in the literature. The best results were observed with tuning classifier parameters as n-estimators $=300$, max-features $=$ sqrt and max-depth $=25$. Generally, for feature space reduction, RF prefers PI over PCA. The model performs exceptionally well for the major classes and improves the macro-level score even when minority classes are not exceptional.

Q-36PI-MLP achieves the highest macro f1-score of 0.81. Chuan long et al. [5] 's study achieved a recall of 0.78 , which is lower compared to those achieved by all evaluated MLP models in this study. For optimal results, the model prefers more features than the rest of the traditional ML models evaluated. The optimal model parameters were set as solver $=$ adam, learning-rate $=$ constant, hidden-layer-sizes $=(200,200,200)$, alpha $=0.0001$ and activation $=$ relu.

Q-26PI-SVM is the best SVM model with a 0.81 macro f1-score. Optimal parameters were identified as kernel $=r b f$, gamma $=7$ and $C=100$. Interestingly, SVM findings exhibit trends different to RF's, in that SVM's recall is significantly better in many cases, and conversely, precision is worse. Furthermore, differences in f1-scores are much lower across other feature selection combinations than RF. This indicates that the SVM is relatively sensitive to features included in the model.

16PI-DT is the best performing DT model with a macro f1-score of 0.76 . The optimal model tuning parameters were min-samples-split $=3$, min-samples-leaf $=3$, max-features $=$ sqrt, max -depth $=25$ and criterion = gini. An opposite model performance effect is observed for scaling and extraction methods. It is observed that Quantile performs better than both Power and Standard when used with 26PCA. Of note, DT preferred fewer features in both validation and these experiments compared to the rest of the models evaluated.

Unlike the rest of the evaluated models, RNN preferred all the features and did not undergo feature space reduction. Due to several factors such as the training approach, loop structure of the neural network, layers, dropout layers, the focus was placed on model tuning. Optimal parameters tuned were observed as epochs $=500$, batch-size $=64$, dropout-rate $=0.2$, learn-rate $=0.001$ and neurons $=128$ compared to learnrate $=0.1$ and neurons $=80$ by Chuan-long et al. [5]. Q-69PIRNN has the best performance at a macro level for detection with an f1-score of 0.73 . The model was effective at anomaly detection due to near-perfect detection rates on major classes compared to the rest of the models but not for the minority classes.

Generally, tuning results show that the CICIDS 2017 dataset yields similar results for Quantile and Power transform methods, where Quantile is typically insignificantly better. Moreover, across the different train/test split ratios, the results show that more training data was not necessarily better in most cases. There is a lack of evidence that an increase in the training data would significantly improve most models. As such, the selection of features in the models was both effective and necessary for real-world adoption.

\subsection{Unmodelled Set Results}

Tables 2 and 3 show unmodelled results for the best CICIDS 2017 classifiers tested against the CICIDS 2018 dataset and the best models for each classifier after retraining with the CICIDS 2018 dataset, respectively. Q-36PI-MLP achieves the best macro f1-score of 0.22 against the CICIDS 2017 dataset. After model retraining, Q-69PI-RNN findings are the best with a macro f1-score of 0.73 and remains similar to those from the previous dataset contrasted with other classifiers. $\mathrm{RF}$ was marginally lower with a $0.72 \mathrm{f} 1$-score with a 0.16 decline against the 0.88 from the first model.

Table 2. CICIDS 2018 dataset against CICIDS 2017 model.

\begin{tabular}{lcccc}
\hline Model & Precision & Recall & $\begin{array}{c}\text { f1-score } \\
\text { (macro) }\end{array}$ & $\begin{array}{c}\text { f1-score } \\
\text { (micro) }\end{array}$ \\
\hline Q-36PI-MLP & 0.22 & 0.25 & 0.22 & 0.81 \\
Q-26PI-SVM & 0.29 & 0.18 & 0.19 & 0.90 \\
Q-26PI-KNN & 0.23 & 0.21 & 0.17 & 0.66 \\
Q-69PI-RNN & 0.14 & 0.15 & 0.13 & 0.78 \\
16PI-DT & 0.08 & 0.13 & 0.09 & 0.62 \\
26PI-RF & 0.06 & 0.07 & 0.06 & 0.81 \\
\hline
\end{tabular}

Table 3. Remodelled CICIDS 2018.

\begin{tabular}{lcc}
\hline Model & $\begin{array}{c}\text { f1-score } \\
\text { (macro) }\end{array}$ & $\begin{array}{c}\text { f1-score } \\
\text { (micro) }\end{array}$ \\
\hline Q-26PCA-MLP & 0.78 & 0.67 \\
Q-26PI-SVM & 0.73 & 0.68 \\
Q-26PI-KNN & 0.77 & 0.66 \\
Q-69PI-RNN & 0.98 & 0.73 \\
16PI-DT & 0.77 & 0.64 \\
26PI-RF & 0.78 & 0.72 \\
\hline
\end{tabular}

All classifiers predict the Benign class with an excellent micro f1-score, greater than 0.80 . The majority classes were detected to some degree by MLP, SVM, KNN and RNN. However, all classifiers failed to predict the minority classes on unmodelled data. Regardless of the low macro performance, MLP, SVM, KNN and RNN models have better detection potential for unmodelled data. These findings from the proposed model address the motivation raised in Section 1, where NIDS are expected to adapt to new scenarios.

An interesting observation is that PCA with a Quantile transform performs well against the CICIDS 2018 dataset. This suggests that PCA may be transforming the data into 
a more uniform distribution. In the table presented, there were no other results of significance observed for the other classifiers.

\section{Conclusion and Future Work}

This paper focused on ML-based NIDS development following a systematic multi-class analysis of classifiers. During the process, an identified challenge was categorising malignant traffic and adapting to novel malicious traffic. In response, several supervised ML classifiers, hyperparameter tuning, feature engineering and data preprocessing activities were explored.

As part of data preprocessing, the effect of Quantile transformation with uniform output on distance-based classifiers - KNN, MLP, SVM and RNN - showed superiority in the macro $\mathrm{f} 1$ of the models. This suggests that a uniform distribution transform to the training dataset inherently benefits the models. The literature reviewed did not identify the need as part of the proposed methodology except for the Standard scaler used by Rosay et al. [11]. In practice, a computer network is likely to follow a uniform distribution in usage, thus likely why the transformation was as effective. Of note, None distance-based classifiers preferred no scaling and had lower accuracy when PCA was applied.

RNN was the best ML classifier in terms of average macro f1-score, followed by RF that performed marginally lower. RNN remains consistent when presented with new datasets, thus reflecting more stability from a macro f1-score perspective. RF does perform better at a micro-class discrimination level to the RNN model. The model is thus a better fit for diverse network traffic classification and novel scenarios compared to the rest of the model evaluated. At a macro level, the rest of the evaluated models perform well.

The macro f1-score was the most reliable metric for this imbalanced dataset as the other metrics - ROC curve, AUC, micro f1-score, accuracy - were hard to distinguish results for analysis. The literature reviewed did not describe the choice of metrics and their effectiveness on models evaluation, to a great extent for minority classes.

PI yielded favourable results over PCA for dimensionality reduction. As part of future work, a correlation matrix and other techniques to identify correlated features during feature selection can be explored to contrast with PI. Given the widespread adoption of the KDD-99 dataset - which has different features extracted to the CICIDS 2017 and CICIDS 2018 datasets used in this paper - additional studies to consolidate features and labelling across datasets can be developed to address limitations that are inherent in model development with different datasets.

\section{Acknowledgments}

This study was funded by National Research Foundation (120654). This work was undertaken in the Distributed Multimedia $\mathrm{CoE}$ at Rhodes University.

\section{References}

[1] Doğukan Aksu, Serpil Ustebay, M.Ali Aydin, and Tulin Atmaca. 2018. Intrusion Detection with Comparative Analysis of Supervised Learning Techniques and Fisher Score Feature Selection Algorithm. Springer International Publishing, Cham, 141-149. https://doi.org/10.1007/9783-030-00840-6_ 16

[2] K. Atefi, H. Hashim, and M. Kassim. 2019. Anomaly Analysis for the Classification Purpose of Intrusion Detection System with K-Nearest Neighbors and Deep Neural Network. In 2019 IEEE 7th Conference on Systems, Process and Control (ICSPC). Institute of Electrical and Electronics Engineers ( IEEE ), USA, 269-274.

[3] Neeraj Bisht and Amir Ahmad. 2017. Analysis of Classifier Ensembles for Network Intrusion Detection Systems. Communications on Applied Electronics 6 (02 2017), 47-53. https://doi.org/10.5120/cae2017652516

[4] Olivier Chapelle, Patrick Haffner, and Vladimir Vapnik. 1999. Support vector machines for histogram-based image classification. IEEE transactions on neural networks / a publication of the IEEE Neural Networks Council 10 (09 1999), 1055-64. https://doi.org/10.1109/72.788646

[5] Yin Chuan-long, Zhu Yue-fei, Fei Jin-long, and He Xin-zheng. 2017. A Deep Learning Approach for Intrusion Detection Using Recurrent Neural Networks. IEEE Access PP (10 2017), 1-1. https://doi.org/10. 1109/ACCESS.2017.2762418

[6] Dorothy E Denning. 1987. An intrusion-detection model. IEEE Transactions on software engineering 1, SE-13 (1987), 222-232.

[7] Michael A. Hayes and Miriam A. M. Capretz. 2014. Contextual anomaly detection framework for big sensor data. Journal of Big Data 2 (2014), $1-22$.

[8] Kurniabudi, D. Stiawan, Darmawijoyo, M. Y. Bin Idris, A. M. Bamhdi, and R. Budiarto. 2020. CICIDS-2017 Dataset Feature Analysis With Information Gain for Anomaly Detection. IEEE Access 8 (2020), 132911132921. https://doi.org/10.1109/ACCESS.2020.3009843

[9] Pramita Muhuri, Prosenjit Chatterjee, Xiaohong Yuan, Kaushik Roy, and Albert Esterline. 2020. Using a Long Short-Term Memory Recurrent Neural Network (LSTM-RNN) to Classify Network Attacks. Information 11 (05 2020), 243. https://doi.org/10.3390/info11050243

[10] Bruno Reis, Eva Maia, and Isabel Praça. 2019. Selection and Performance Analysis of CICIDS2017 Features Importance. In International Symposium on Foundations and Practice of Security. Springer, Springer International Publishing, Cham, 56-71.

[11] Arnaud Rosay, Florent Carlier, and Pascal Leroux. 2020. MLP4NIDS: An Efficient MLP-Based Network Intrusion Detection for CICIDS2017 Dataset. In Machine Learning for Networking, Selma Boumerdassi, Éric Renault, and Paul Mühlethaler (Eds.). Springer International Publishing, Cham, 240-254.

[12] Bruce Schneier. 2015. Secrets and lies: digital security in a networked world (1st ed.). John Wiley \& Sons, Inc., USA.

[13] Iman Sharafaldin., Arash Habibi Lashkari., and Ali A. Ghorbani. 2018. Toward Generating a New Intrusion Detection Dataset and Intrusion Traffic Characterization. In Proceedings of the 4th International Conference on Information Systems Security and Privacy ICISSP,. INSTICC, SciTePress, Canada, 108-116. https://doi.org/10. 5220/0006639801080116

[14] Chih-Fong Tsai, Yu-Feng Hsu, Chia-Ying Lin, and Wei-Yang Lin. 2009. Intrusion detection by machine learning: A review. expert systems with applications 36, 10 (2009), 11994-12000. 